\title{
Signal and response properties indicate an optoacoustic effect underlying the intra-cochlear laser- optical stimulation
}

Nicole Kallweit, Peter Baumhoff, Alexander Krueger, Nadine Tinne, Alexander Heisterkamp, et al.

Nicole Kallweit, Peter Baumhoff, Alexander Krueger, Nadine Tinne, Alexander Heisterkamp, Andrej Kral, Hannes Maier, Tammo Ripken, "Signal and response properties indicate an optoacoustic effect underlying the intracochlear laser-optical stimulation," Proc. SPIE 9689, Photonic Therapeutics and Diagnostics XII, 96892G (1 March 2016); doi: 10.1117/12.2210926

SPIE. Event: SPIE BiOS, 2016, San Francisco, California, United States 


\title{
Signal and response properties indicate an optoacoustic effect underlying the intra-cochlear laser-optical stimulation
}

\author{
Nicole Kallweit* ${ }^{\mathrm{a}, \mathrm{d}}$, Peter Baumhoff ${ }^{\mathrm{b}}$,Alexander Krueger ${ }^{\mathrm{a}, \mathrm{d}}$, Nadine Tinne ${ }^{\mathrm{a}}$, \\ Alexander Heisterkamp ${ }^{\mathrm{a}, \mathrm{c}, \mathrm{d}}$, Andrej Kral ${ }^{\mathrm{b}, \mathrm{d}}$, Hannes Maier ${ }^{\mathrm{b}, \mathrm{d}}$, Tammo Ripken ${ }^{\mathrm{a}, \mathrm{d}}$ \\ ${ }^{a}$ Laser Zentrum Hannover e.V., Hollerithallee 8, 30419 Hannover, Germany; ${ }^{b}$ Institute of \\ Audioneurotechnology and Dept. of Experimental Otology, ENT Clinics, Hannover Medical School, \\ Feodor-Lynen-Str. 35, 30625 Hannover, Germany; 'Leibniz University Hannover, Welfengarten 1, \\ 30167 Hannover, Germany; ${ }^{\mathrm{d} C l u s t e r}$ of Excellence "Hearing4all"
}

\begin{abstract}
Optical cochlea stimulation is under investigation as a potential alternative to conventional electric cochlea implants in treatment of sensorineural hearing loss. If direct optical stimulation of spiral ganglion neurons (SGNs) would be feasible, a smaller stimulation volume and, therefore, an improved frequency resolution could be achieved. However, it is unclear whether the mechanism of optical stimulation is based on direct neuronal stimulation or on optoacoustics. Animal studies on hearing vs. deafened guinea pigs already identified the optoacoustic effect as potential mechanism for intra-cochlear optical stimulation.

In order to characterize the optoacoustic stimulus more thoroughly the acoustic signal along the beam path of a pulsed laser in water was quantified and compared to the neuronal response properties of hearing guinea pigs stimulated with the same laser parameters. Two pulsed laser systems were used for analyzing the influence of variable pulse duration, pulse energy, pulse peak power and absorption coefficient.

Preliminaryresults of the experiments in water and in vivo suggesta similar dependency of response signals on the applied laser parameters: Both datasets show an onset and offset signal at the beginning and the end of the laser pulse. Further, the resulting signal amplitude depends on the pulse peak power as well as the temporal development of the applied laser pulse. The data indicates the maximum of the first derivative of power as the decisive factor. In conclusion our findingsstrengthen the hypothesis of optoacoustics as the underlying mechanism for optical stimulation of the cochlea.
\end{abstract}

Keywords: Laser, optoacoustic effect, optical stimulation, cochlea, hearing loss, infrared neural stimulation

\section{INTRODUCTION}

In recent years many studies and reports have been published about the optical stimulation of various nerves ${ }^{1-6}$. For this reason, the optical stimulation of the cochlea as a higher resolved and artifact-free method to conventional cochlear implants is discussed.

However, the stimulation mechanism is unclear and is controversially discussed in literature. The optical intra-cochlear stimulation is based on direct activation of the spiral ganglion neurons ${ }^{7-11}$ or on the optoacoustic effect ${ }^{12-16}$. The optoacoustic mechanism deflects the basilarmembrane of the cochlea and activates the inner hair cells. This hypothesis is supported by measurements of laser pulse activated movements of the basilarmembrane ${ }^{17-19}$. The distinction between infrared neural stimulation (INS) and the optoacoustic mechanism is necessary for future applications. In this study, in vivo measurements of compound action potentials (CAPs) were compared with pressure measurements in water. To better understand the optical stimulation of the cochlea the same laser parameter were used in both settings. The pulse duration was varied from nanoseconds to milliseconds and the wavelength was kept constant with, firstly, a constant pulse energy and, secondly, a constant pulse peak power. The experiments were performed in thermal confinement, stress confinement as well as outside of both confinements ${ }^{20}$ in order to verify the hypotheses that CAPs can be generated by the optoacoustic mechanism in hearing animals.

*n.kallweit@1zh.de; phone +49 511 2788-225; fax +49 511 2788-100; lzh.de

Photonic Therapeutics and Diagnostics XII, edited by Bernard Choi, et al., Proc. of SPIE Vol. 9689, 96892G · C 2016 SPIE · CCC code: 1605-7422/16/\$18 · doi: 10.1117/12.2210926 


\section{MATERIALS AND METHODS}

\subsection{In vivo}

All in vivo experiments have been performed in accordance to the animal welfare guidelines of Germany and the European Union. All experimental procedures were approved by the German state authorities and the animal welfare officer of the research facilities.

The measurements were performed with normal hearing (threshold $<30-40 \mathrm{~dB}$ sound pressure level) Dunkin Hartley guinea pigs ( $\mathrm{n}=8$, Charles River) of either sex anesthetized by a solution of ketamine and xylazine. To measure CAPs a silver ball electrode was positioned in the round window niche in contact to the round window membrane (RWM). The CAP signal was filtered (Butterworth filter 6th order, high-pass frequency $5 \mathrm{~Hz}$, low-pass frequency $5 \mathrm{kHz}$ ) and amplified by $80 \mathrm{~dB}$. The optical fiber was placed in the perilymph of the scala tympani through a cochleostomy. Before and after surgery the hearing function was tested by measuring click-evoked auditory brainstem responses (ABRs).

\subsection{Hydrophone measurements}

A hydrophone (8103, Brüel\&Kjær Sound \& Vibration Measurement A/S, Denmark) was used for the investigation of the optoacoustic signal generation. To measure the pressure at the fiber tip, the hydrophone was placed horizontally inside a water-filled cylinder $(\mathrm{h}=194 \mathrm{~mm}, \varnothing=194 \mathrm{~mm}, \mathrm{~V}=5.7 \mathrm{l})$ and the fiber was placed orthogonally with a distance of $0.1 \mathrm{~mm}$ to the hydrophone. The signal was amplified (Nexus 2692-0S1 bandwidth of $100 \mathrm{kHz}$, Brüel\&Kjær Sound \& Vibration Measurement A/S, Denmark) and averaged over ten thousand laser pulses to improve the signal to noise ratio (SNR). The acrylic cylinder was filled with water, which is the main component of perilymph ${ }^{21}$.

\subsection{Laser systems}

Two laser systems were used to analyze the stimulation mechanism in thermal confinement as well as stress confinement condition. The first laser (Capella, Lockheed Martin Aculight, WA, USA) had a fixed wavelength of $1860 \mathrm{~nm}$, a variable pulse duration from $10 \mu \mathrm{s}$ up to $20 \mathrm{~ms}$ and a repetition rate between $2.5 \mathrm{~Hz}$ and $100 \mathrm{~Hz}$ depended on the pulse duration.

The second laser (Ekspla, NT342A, Vilnius, Lithuania) was a tunable system with an optical parametric oscillator (OPO). The pulse duration was fixed at $4 \mathrm{~ns}$, the repetition rate at $10 \mathrm{~Hz}$ and the wavelength could be adjusted between $420 \mathrm{~nm}$ and $2300 \mathrm{~nm}$. The lasers were coupled into a multimode fiber (FG105LCA-CUSTOM, $105 \mu \mathrm{m}$ core diameter, NA 0.22 , low $\mathrm{OH}$, Thorlabs, NJ, USA) for the experiments.

Two series of experiments were performed: The first with a constant pulse energy of $6 \mu \mathrm{J}$, a constant wavelength of $1860 \mathrm{~nm}$ and a variable pulse duration between $5 \mathrm{~ns}$ up to $200 \mu \mathrm{s}$. The second parameter study was performed with a constant pulse peak power of $150 \mathrm{~mW}$, a constant wavelength of $1860 \mathrm{~nm}$ and a variable laser pulse duration between $10 \mu \mathrm{s}$ up to $10 \mathrm{~ms}$. The in vivo experiments as well as the pressure measurements were conducted with the same laser systems and parameters. The temporal laser pulse form for pulse durations longer or equal than $10 \mu \mathrm{s}$ (Fig. 1, top) was measured with an InGaAs detector (DET10D, Thorlabs, NJ, USA) with a rise time of $25 \mathrm{~ns}$. The first derivative of the pulse power was calculated numerically by averaging forward and backward derivative (Fig. 1, bottom).

\section{RESULTS}

For the constant pulse energy of $6 \mu \mathrm{J}$, the CAP amplitude decreases with increasing pulse duration (Fig. 2a, top). For longer pulse duration than $200 \mu \mathrm{s}$, no CAP signal can be detected (data not shown). The first CAP signal in figure 2a (top) was generated with the $5 \mathrm{~ns}$ laser pulse of the Ekspla laser in stress confinement condition, the other signals were performed with the Capella laser in thermal confinement. The signal amplitude of the pressure measurements showed the same behavior as the CAP amplitude. The pressure amplitude decreases with an increasing pulse duration (Fig. 2a, center). Here, the maximum of the first derivative of power can only be measured for laser pulses greater or equal than $10 \mu \mathrm{s}$. Therefore, the first value of the first derivative of power cannot be calculated and is missing (Fig. 2a, bottom). The maximum of the first derivative decreases with an increasing laser pulse duration (Fig. 2a, bottom) similar to the CAP amplitude and the pressure amplitude. This signal behavior might be explained by the pulse peak power. If the pulse energy is constant and the pulse duration increases, the pulse peak power will decreases. Therefore, the following measurements were performed with a constant pulse peak power. It was kept constant at $150 \mathrm{~mW}$ and the laser pulse duration was varied. The CAP amplitude was nearly constant (Fig. 2b, top) except for very short pulse durations. In contrast to the CAP data, the pressure signal amplitude was constant for all laser pulse durations for the fixed pulse peak 
power (Fig. 2b, center). The maximum of the first derivative of power was steady (Fig. 2b, bottom) similar to the pressure signal.

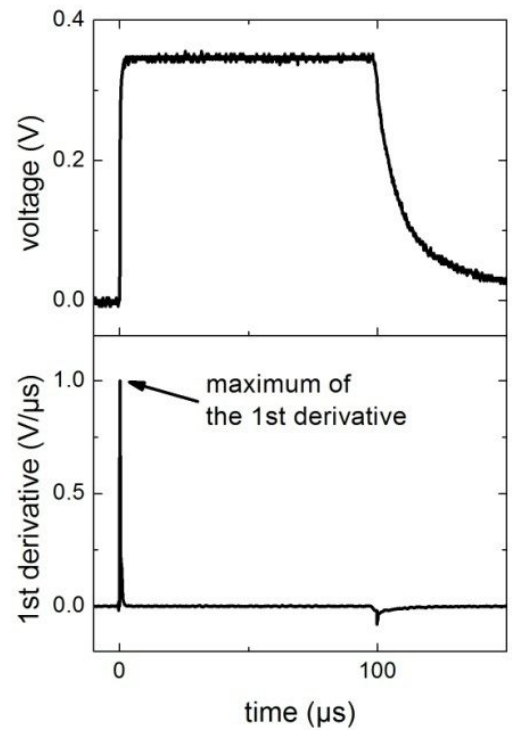

Figure 1. The temporal laser pulse form for a laser pulse duration of $100 \mu \mathrm{s}$ (top) measured by a photodiode and the first derivative of this laser pulse power (bottom). The maximum of the first derivative is marked (bottom).
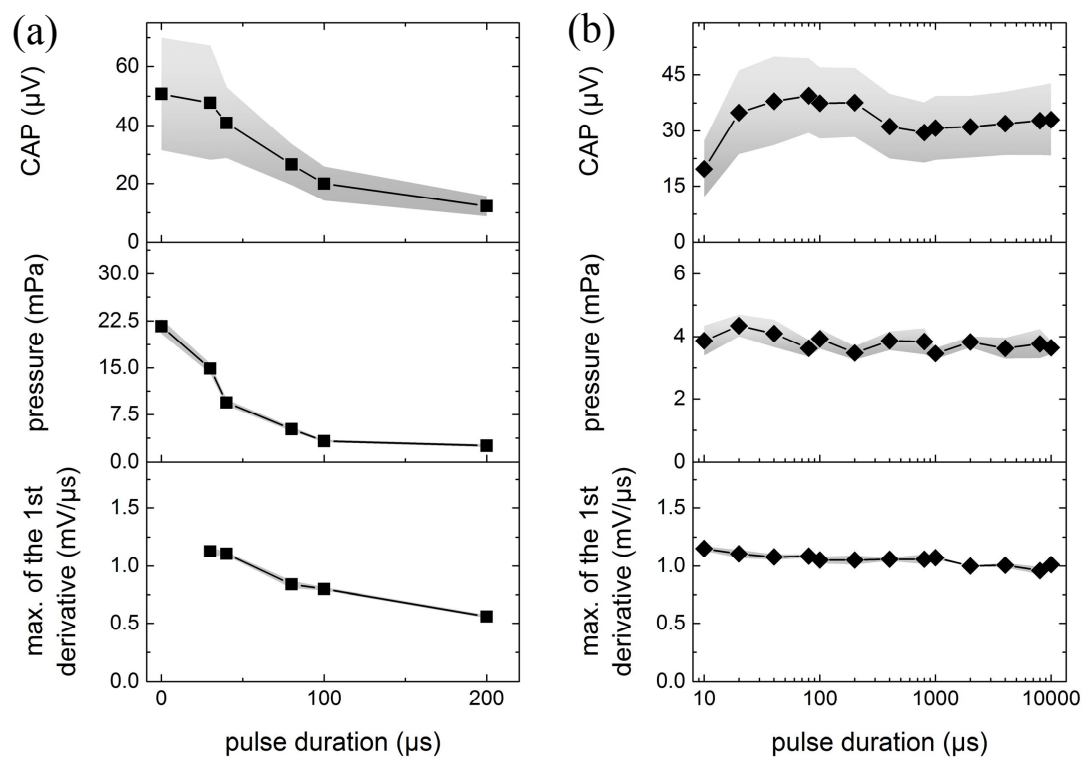

Figure 2. (a) CAP amplitude (top, $\mathrm{n}=8$ ), pressure amplitude measured by a hydrophone (center, $\mathrm{n}=5$ ) and the maximum of the first derivative of power as a function of laser pulse duration for constant pulse energy. The first value was performed with the Ekspla laser at pulse duration of $5 \mathrm{~ns}$, the other with the Capella laser. Here, the temporal laser pulse form could not be measured for laser pulses $\tau \leq 10 \mu \mathrm{s}$. Therefore, the first value of the first derivative of power cannot be calculated and is missing (bottom). (b) CAP (top, $\mathrm{n}=8$ ), pressure amplitude (center, $\mathrm{n}=5$ ) and the maximum of the first derivative as a function of pulse duration for constant pulse peak power of $150 \mathrm{~mW}$. Here, for the constant pulse peak power the condition fulfilled only thermal confinement. 
For the pulse peak power of $150 \mathrm{~mW}$ two pressure pulse can be detected (Fig. 3, left). The separated signal onset and offset have the time interval of the applied laser pulse duration. In in vivo measurements, separate CAP signals were observed for pulse durations longer than $1 \mathrm{~ms}$ (Fig. 3, right). For laser pulse durations less than or equal $1 \mathrm{~ms}$ only one single CAP signal can be measured.
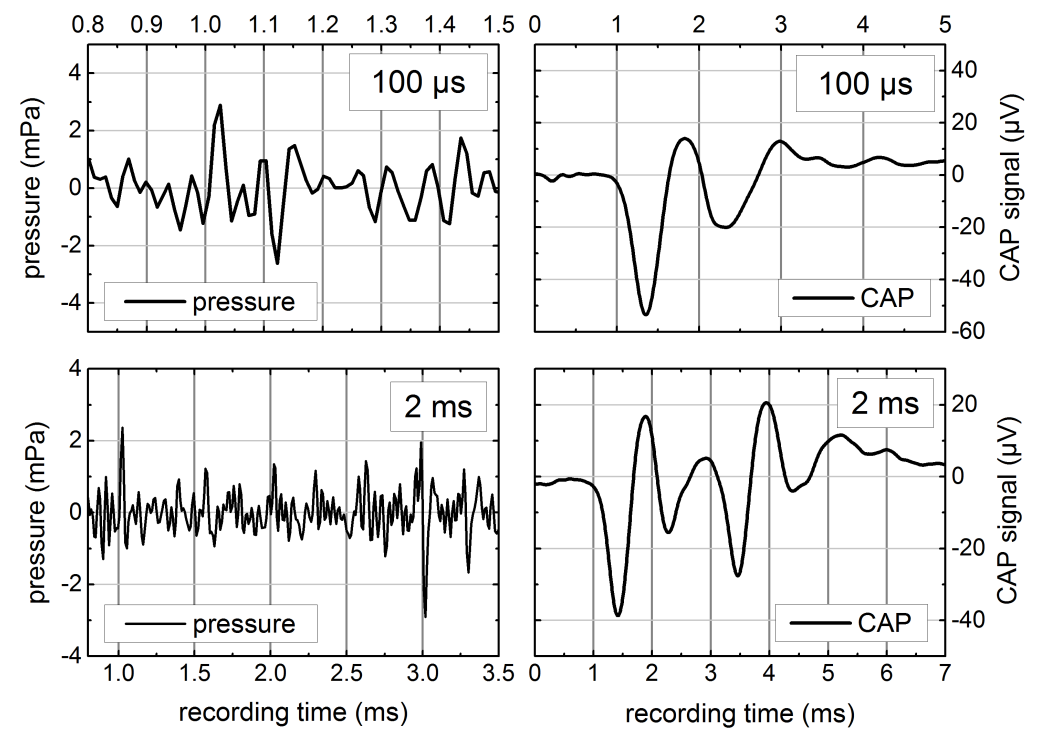

Figure 3. Selected pressure amplitudes measured using a hydrophone (left) show an onset and offset response. CAPs (right) show an onset and offset response for pulse durations longer than $1 \mathrm{~ms}$. The delay was $1 \mathrm{~ms}$ for all measurements.

\section{DISCUSSION}

The findings indicate that optoacoustic is the basic mechanism for optical cochlear stimulation. The signal amplitude behavior of the pressure measurements and in vivo data were the same. The volume of the water-filled cylinder $(\mathrm{V}=61)$ and of the cochlea $\left(\mathrm{V}=\sim 10 \mu 1^{22}\right)$ were completely different, but the resonance of the cylinder was not relevant for the analysis of the signal amplitude. Water was used instead of perilymph for the pressure measurements in the physical model, because, firstly, water is the main component of the perilymph ${ }^{21}$ and, secondly there is no relevant absorption by the proteins of the perilymph in the spectral range of the used laser which works far off the electronic absorptions in the UV-range ${ }^{23}$. The observed signal with its characteristic onset and offset peaks strongly supports the hypotheses of optoacoustic, because the time between the beginning and the end of the response for in vivo and for pressure measurements are equal to the laser pulse duration. The fact that two CAP signals were only detectable for pulse durations longer than or equal to $2 \mathrm{~ms}$, can be explained by the prolonged integration time of the compound auditory nerve response. The fact, that no pressure wave between the onset and offset signal can be measured, can be explained by the physics of optoacoustics, where constant heating generates no pressure wave $\mathrm{e}^{24}$. Thus, the amplitude depends on the pulse peak power and the maximum of the first derivative of power. On the basis of these findings, doubt exists on direct optical stimulation of SGNs as the basic mechanism in experiments performed in normal hearing animals or in acutely deafened animals with some degree of residual hearing.

\section{CONCLUSION}

The similarity of the pressure measurements in water and the in vivo CAP measurements supports the hypothesis of optoacoustic as the basic mechanism for optical cochlear stimulation. Based on the current results of this study, and on previous experiments of deafened cochleae which did not show any response to optical stimulation, we conclude that pressure sensitive hair cells are necessary and direct infrared neural stimulation of neuronal ganglion cells in the cochlea 
does not work. Further analysis of the results and a more detailed description of the spectral absorption characteristics and optoacoustic phenomena underling the signals will be published soon.

\section{ACKNOWLEDGMENTS}

This work was supported by the DFG Cluster of Excellence EXC 1077/1 "Hearing4all", in part by MedEl Comp., Innsbruck, Austria and EU grant ACTION (FP7 Project No. 611230; P. Baumhoff and A. Kral).

\section{REFERENCES}

[1] Tozburun, S., Lagoda, G. a., Burnett, A. L., Fried, N. M., "Subsurface near-infrared laser stimulation of the periprostatic cavernous nerves," J. Biophotonics 5, 793-800 (2012).

[2] Jenkins, M. W., Duke A. R., Gu S., Doughman Y., Chiel H. J., Fujioka H., Watanabe M., Jansen E. D., Rollins A. M., "Optical pacing of the embryonic heart," Nat. Photonics 4, 623-626 (2010).

[3] Duke, A. R., Cayce J. M., Malphrus J. D., Konrad P., Mahadevan-Jansen A., Jansen E. D., "Combined optical and electrical stimulation of neural tissue in vivo," J. Biomed. Opt. 14, 2009-2011 (2009).

[4] Fried, N. M., Lagoda, G. a, Scott, N. J., Su, L.-M., Burnett, A. L., "Laser stimulation of the cavernous nerves in the rat prostate, in vivo: optimization of wavelength, pulse energy, and pulse repetition rate," Conf. Proc. IEEE Eng. Med. Biol. Soc. 2008, 2777-2780 (2008).

[5] Wells, J., Kao, C., Konrad, P., Milner, T., Kim, J., Mahadevan-Jansen, A., Jansen, E. D., "Biophysical mechanisms of transient optical stimulation of peripheral nerve," Biophys. J. 93, 2567-2580 (2007).

[6] Gimeno, M. A., Roberts, C. M., Webb, J. L., "Acceleration of Rate of the Early Chick Embryo Heart by Visible Light." Nature 214, 1014-1016 (1967).

[7] Tan, X., Young, H., Matic, A.I., Zirkle, W., Rajguru, S, Richter, C.-P., "Temporal properties of inferior colliculus neurons to photonic stimulation in the cochlea," Physiol. Rep. 3, e12491 (2015).

[8] Shapiro, M. G., Homma, K., Villarreal, S., Richter, C.-P., Bezanilla, F., "Infrared light excites cells by changing their electrical capacitance," Nat. Commun. 3, 736 (2012).

[9] Richter, C.-P., Rajguru, S. M., Matic, A. I., Moreno, E. L., Fishman, A. J., Robinson, A. M., Suh, E., Walsh, J. T., "Spread of cochlear excitation during stimulation with pulsed infrared radiation: inferior colliculus measurements," J. Neural Eng. 8, 056006 (2011).

[10] Rajguru, S. M., Matic, A. I., Robinson, A. M., Fishman, A. J., Moreno, L. E., Bradley, A., Vujanovic, I., Breen, J., Wells, J. D., Bendett, M., Richter, C.-P., "Optical cochlear implants: Evaluation of surgical approach and laser parameters in cat,." Hear. Res. 269, 102-111 (2010).

[11] Izzo, A. D., Walsh, J. T., Ralph, H., Webb, J., Bendett, M., Wells, J., Richter, C.-P., "Laser stimulation of auditory neurons: effect of shorter pulse duration and penetration depth," Biophys. J. 94, 3159-3166 (2008).

[12] Thompson, A. C., Fallon, J. B., Wise, A. K., Wade, S. A., Shepherd, R. K., Stoddart, P. R., "Infrared neural stimulation fails to evoke neural activity in the deaf guinea pig cochlea," Hear. Res. 324, 46-53 (2015).

[13] Rettenmaier, A., Lenarz, T., Reuter, G., "Nanosecond laser pulse stimulation of spiral ganglion neurons and model cells," Biomed. Opt. Express 5, 1014 (2014).

[14] Schultz, M., Baumhoff, P., Kallweit, N., Sato, M., Krüger, A., Ripken, T., Lenarz, T., Kral, A., "Optical stimulation of the hearing and deaf cochlea under thermal and stress confinement condition," Proc. SPIE 8928 (In: Hirschberg, H. et al.), 892816 (2014).

[15] Schultz, M., Baumhoff, P., Maier, H., Teudt, I. U., Krüger, A., Lenarz, T., Kral, A., "Nanosecond laser pulse stimulation of the inner ear-a wavelength study," Biomed. Opt. Express 3, 3332-45 (2012).

[16] Teudt, I. U., Maier, H., Richter, C. P., Kral, A., "Acoustic events and 'optophonic' cochlear responses induced by pulsed near-infrared LASER," IEEE Trans. Biomed. Eng. 58, 1648-1655 (2011).

[17] Wenzel, G. I., Lenarz, T., Schick, B., "Welche Farben könnten wir hören?" HNO 62, 82-87 (2014).

[18]Zhang, K. Y., Wenzel, G. I., Balster S., Lim, H. H., Lubatschowski, H., Lenarz, T., Ertmer, W., Reuter, G., "Optoacoustic induced vibrations within the inner ear," Opt. Express 17, 23037-23043 (2009).

[19] Fridberger, A., Ren, T., "Local mechanical stimulation of the hearing organ by laser irradiation," Neuroreport 17, 33-37 (2006). 
[20] Paltauf, G., "Photoacoustic waves excited in liquids by fiber-transmitted laser pulses," J. Acoust. Soc. Am. 104, 890 (1998).

[21] Cole, B. J. D., Richard, S., "An Approach to Mechanics of the Cochlea," J. of Applied Mathematics and Physics $28,(2000)$

[22] Thorne, M., Salt, A. N., Demott, J. E., Henson, M. M., Henson, O. W., Gewalt, S. L., "Cochlear Fluid Space Dimensions for Six Species Derived From Reconstructions of Three-Dimensional Magnetic Kesonance lmages, " The Laryngoscope 10, 1661-1668 (1999).

[23] Goldfarb, R., Saidel, L. j., Mosovich, E., "The ultraviolet absorption spectra of proteins," J. Biol. Chem. 193, 397-404 (1951).

[24] Wang, L. V., "Tutorial on Photoacoustic Microscopy and Computed Tomography," IEEE 14, 171-179 (2008). 